\title{
CONFERENCE HANDBOOK
}

\section{INTERNATIONAL}

\section{CONFERENCE ON \\ LOW TEMPERATURE PHYSICS}

Sponsored by the International Union of Pure and Applied Physics

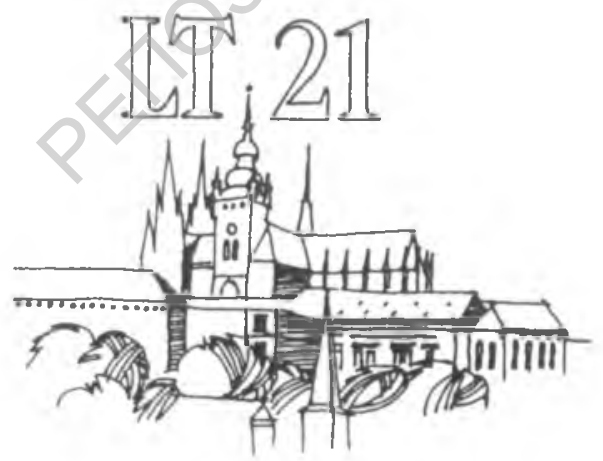

August $8-14,1996$

Prague, Czech Republic 
AEP $\bar{T}$ Magnetic non-linearity at strong Hall drift

VR. Sobol and B.B. Boiko

Institute of Solid State and Semiconductor Physics, BAN, P.Brovka str.17, 220072, Minsk, Belarus

Steady current non-linear phenomena connected with the dependence of conductivity tensor components on self magnetic field of Hall drift have been investigated in aluminum polycrystalline conductors. Strong Hall drift has been arranged by applying of cylinder conductor geometry, transport current being made to flow between inner and outer concentric contacts. Self magnetic field and resistivity are studied as a function of external field, transport current densities and temperature. The self magnetic field magnitude in this geometry achieves the levels of external field. The role of dispersion law anisotropy and elongated electron orbits are analysed by the method of orientation and kinetic averaging of conductivity tensor during charge diffusion. The voltage stabilization observed at heat generation near helium boiling crysis Indicates the possibility of existence of spatial electric-temperature structures.

AEP8 Electrical conductivity and magneto-resistance studies on polyaniline-PMMA blends at very low temperature

D. Srinivasan “, Anasuya Raghunathan ", T.S. Natarajan *, G. Rangarajan ", C.K. Subramaniam" , and $B$. Wessling ${ }^{e}$

-Department of Physics, Indian Institute of Technology, Madras 600036

'Center for Electrochemical \& Energy research, SPIC Science Foundation, 110, Mount road, Madras 32

'Zipperling Kessler and Co., Postfach 1464,D-22904, A hrensburg, Germany

dDepartment of Physics, Indian Institute of Science, Bangalore 560012

D.C. electrical conductivity of polyaniline $(33 \%, 40 \%)$ blended with PMMA was measured from 5K to $300 \mathrm{mK}$. The conductivity behavior is consistent with fluctuation induced tunneling. Magneto-resistance (MR) was measured between $300 \mathrm{~K}$ and $2 \mathrm{~K}$. From $20 \mathrm{~K}$ to $2 \mathrm{~K}_{1}$ a large positive MR was observed. At $2 \mathrm{~K}$, for low magnetic fields( $<1$ Tesla), a deviation from the normal $\mathbf{H}^{2}$ behaviour was observed.

\section{AEP9 Effect of nuclear field on magnetotransport quantum oscillations in InSb}

N. Gauss and A.G.M. Jansen

Grenoble High Magnetic Field Laboratory, MPI-FKF and CNRS, B.P. 166, F-38042 Grenoble, France

We measure the hyperfine contribution on resistance and Hall-voltage in the quantum limit of metallically doped $\mathrm{n}-\mathrm{InSb}$ in combining a transport measurement with NMR. Sweeping a radio frequency signal over the nuclear resonances (fast adiabatic frequency passage) in our sample inverses the nuclear magnetization thereby inducing a change in $R_{x x}$ and $R_{x y}$. In this way we study the dependence of the electronic density of states on the hyperfine field. 